\title{
Designing Glitch Procedures and Visualisation Workflows for Markerless Live Motion Capture of Contemporary Dance
}

\author{
Stephan Jürgens \\ ITI/LARSyS \\ Funchal, Portugal \\ stephan.jurgens@iti.larsys.pt
}

\author{
Nuno N. Correia \\ University of Greenwich \\ London, UK \\ ITI/LARSyS \\ Funchal, Portugal \\ n.correia@greenwich.ac.uk
}

\author{
Raul Masu \\ ITI/LARSyS \\ Funchal, Portugal \\ FCT - NOVA University of Lisbon \\ Lisbon, Portugal \\ raul.masu@iti.larsys.pt
}

\begin{abstract}
This paper presents a case study in the exploration and creative usage of errors and glitches in the real-time markerless motion capture of contemporary dance. We developed a typology of MoCap failures comprised of seven categories, allowing the user to situate each distinct error in the respective stage of the motion capture pipeline. This way, glitch procedures for the creative use of 'bad' MoCap data were designed, resulting in uncommon avatar visualisations. We propose an additional 're-visualisation' module in our motion capture pipeline and avatar staging approach, which enables choreographers and digital artists to rapidly prototype their ideas in a mixed reality performance environment. Finally, we discuss how our extended MoCap pipeline and avatar staging set-up can support artists and researchers who aim at a flexible and adaptive workflow in real-time motion visualization.
\end{abstract}

\section{CCS CONCEPTS}

- Applied computing $\rightarrow$ Performing arts; • Computing methodologies $\rightarrow$ Motion capture; $\bullet$ Human-centered computing $\rightarrow$ Empirical studies in interaction design;

\section{KEYWORDS}

Motion capture, glitch, digital error, markerless, live visuals, contemporary dance, mixed reality set-up, visualisation, choreography

\section{INTRODUCTION}

Live motion capture systems have become more accessible to artists and researchers during the past decade. In particular, markerless motion capture offers quick set-up times and unobtrusive stage presence, not to mention the much lower price point of these systems. To work with markerless live motion capture in contemporary dance, both in rehearsal and performance, provides exciting opportunities to explore real-time motion visualisations, combining physical and virtual dimensions of reality.

In our paper we will use the term 'mixed reality set-up' in accordance with previous work in the field of Human-Computer Interaction (HCI) by Gagneré and Plessiet [4]. These authors' use of the term parallels media theorist Chris Salter's definition of mixed reality: "Interdisciplinary research area examining the hybrid interaction of physical and virtual elements (e.g. computer generated graphics) together in the same space" [10]. Additionally, we will employ the term 'mixed reality performance environment,' which clarifies where and for what purposes a 'mixed reality set-up' is used.

This paper examines both the technical limits of the markerless motion capture system available to us, and the impact of distinct performance techniques (e.g. improvisational tasks carried out by the dancers) on the performance of the system and the real-time motion visualisations. Our analysis of this combination of physical and virtual dimensions in the performance environment of our case study allows us to make contributions for future research and artistic work with markerless motion capture systems in mixed reality performance environments.

The case study presented in this paper has been carried out in the context of the Moving Digits ${ }^{1}$ project. During the initial stages of the project, a group of ten dancers and choreographers explored prototypes of interactive visuals developed by the research team (including the first and third authors of this paper), based on ideas expressed by the artists in an initial focus group session [6]. Subsequently, a call for artistic projects with these prototypes resulted in the selection of four participants' projects. These projects were then be developed over a two-week artistic residency. One of the selected projects was The Beautiful Glitch by Portuguese choreographer Sylvia Rijmer, which serves as our case study here.

The main objective of Rijmer's project proposal was to specifically explore glitches, errors and failure working with the motion capture system available during the artistic residency. Over the past years, Rijmer has developed the Body Logic Method (BLM), which can be best described as a choreographic system consisting of improvisational techniques and compositional principles for contemporary dance. Our case study shows: how Rijmer has used BLM to work with dancers in a mixed reality performance environment;

${ }^{1}$ https://movingdigits.eu 
which results were achieved; and what was presented in the public performance of the project. The case study also shows how we adapted our technical set-up and collaboration methods to support the artist's vision and needs.

Our field work for this case study was conducted during a twoweek artistic residency at $\mathrm{STL}^{2}$, Tallinn. Rijmer had two local dancers to work with. A dedicated real-time markerless motion capture (MoCap) system (The Captury) ${ }^{3}$ was set up and available throughout the residency. MoCap data was fed into Unity $3 \mathrm{D}^{4}$ and visualised by means of 3D avatars. These were sent as a video stream via NDISyphon ${ }^{5}$ software to Isadora $^{6}$, a programming platform for live interactive audiovisuals in performance and installations. Technical support was provided by digital artists and researchers from the team of Moving Digits.

Methodologically, we adopted a multimodal approach to documenting in the field. We aimed to collect information about the actual context of use, as proposed in the 'technomethodology' by Dourish, gathering: "Fundamental insights about the organisation of the action being moment-to-moment, naturally occurring, improvisational response to practical problems" [3]. To this end, we combined different sources: photography and video recordings of the rehearsals and public showcase were collected; notebooks of dancers, choreographers and technicians were photographed; interviews with the choreographers were conducted after each rehearsal; and versions of the software files were kept after each change.

For analysis, interpretation and discussion of our data, we draw on theories and literature from different academic fields and disciplines. In media art literature, the topics of glitch art and glitch studies have been explored. Useful classifications of error and failure are suggested, which serve as a theoretical lens for our case study. In the field of HCI we find literature to contextualize Rijmer's work, and to discuss real-time MoCap, in mixed reality set-ups and interactive visualisations in performance.

\section{LITERATURE REVIEW}

\subsection{Glitch, Glitch Art and Glitch Studies}

A central reference regarding the concept of glitch in the context of the arts is Kim Cascone's article "The Aesthetics of Failure," in which he provides a brief history of glitch in music and suggests a typology of unexpected computational failures, comprised of "glitches, bugs, application errors, system crashes, clipping, aliasing, distortion, quantisation noises, and even the noise floor of computer sound cards" [2]. For Cascone, these types of failure in digital technology have become materials that composers can incorporate in their creative process. In doing so, much recent artistic work has emerged and constituted a unique 'aesthetics of failure'. Since meaning is de-constructed and re-constructed in such artistic practice, "the listener takes an active role in the production of meaning" [2].

Mark Nunes looks at artistic "strategies of misdirection" and advocates that error suggests "ways in which failure, glitch and miscommunication provide creative openings and lines of flight

\footnotetext{
${ }^{2}$ https://stl.ee

${ }^{3}$ https://thecaptury.com/

${ }^{4}$ https://unity.com/

${ }^{5}$ https://docs.vidvox.net/freebies_ndi_syphon.html

${ }^{6}$ https://troikatronix.com/
}

that allow for a reconceptualisation of what can (or cannot) be realized within existing social and cultural practice" [9]. What's more, error can evidence a system's failure as much as reveal its (usually invisible) operational logic.

Looking at Glitch Art from a visual arts perspective, Michael Betancourt links Cascone's types of failures to glitch practices in computer graphics and digital imaging techniques. He sees a parallel to experimental film and expanded cinema, both historical avantgarde practices, which made the materiality of the photographic (hence also film) medium visible through focussing on the operation (and malfunction) of camera, lenses, film graininess, flicker and so forth [1]. For Betancourt, "resolution, compression, artifacts - as well as technical errors of all types in any reproduction technology" can be seen as corresponding glitch procedures to Cascone's list [1]. Through revealing the materiality of digital media, the artist can "violate the aura of the digital," i.e. expose "the illusion of a self-productive domain, infinite, capable of creating value without expenditure, unlike the reality of limited resources, time, expense, etc. that otherwise govern all forms of value and production" [1]. In other words, the use of glitch procedures has the potential for critique, to be a political gesture. In Betancourt's view, critical media practice and the making-conscious entirely depend on how glitch procedures are employed by the artist, who may as well decide to work with the glitch for aesthetic reasons only.

Betancourt's stance resonates with Rosa Menkman's "Glitch Studies Manifesto" [7]. Menkman states: "This 'new' form of 'conservative glitch art' or 'hot glitch art' focuses more on design and end products than on the procedural and political breaking of flows. There is an obvious critique: to design a glitch means to domesticate it" [7]. Distinguishing between glitch art and the experience of a glitch, Menkman suggests: "The glitch has no solid form or state through time; it is often perceived as an unexpected and abnormal modus operandi, a break from (one of) the many flows (of expectations) within a technological system" [7]. Since for Menkman "the procedural essence of glitch art is opposed to conservation," (as in 'conservative glitch art'), she instead proposes 'glitch studies,' a working method that allows to balance technological progression with its inherent accident. Central to glitch studies as proposed by Menkman is "the search for the unfamiliar while at the same time it tries to de-familiarize the familiar" [7].

\subsection{Experimentation with Glitch Procedures in Markerless Motion Capture Set-Ups}

A. Bill Miller has recorded noise generated by motion capture data (digital mistakes), which was acquired with Organic Motion ${ }^{7}$, a markerless MoCap system [8]. Rather than "cleaning" the noise in a traditional way, Miller analysed the causes of the digital error to understand the conditions in which glitches occured in their MoCap system. The recorded glitch data was then connected to 3D modeled shapes for abstract animations; e.g. jittery MoCap data were visualised by means of typographic characters parented to bones, or noise data was mapped to 3D cloth simulations. Miller's theoretical perspective and capture methods are highly relevant for our case study - however, we will focus on real-time motion

${ }^{7}$ https://tracklab.com.au/organic-motion/ 
capture and data visualisations with avatars of human shape in a mixed reality performance environment.

We detect a gap in HCI literature regarding glitch procedures in real-time markerless motion capture as part of a mixed reality performance environment. We suggest adopting a 'glitch studies' approach and reference relevant work that describes aspects of wellfunctioning real-time motion capture in mixed reality performance environments as a theoretical base to look at the glitch procedures in our case study.

Joe Geigel and Marla Schweppe have looked at the motion capture pipeline for real-time control of virtual actors [5]. They identify three main modules in their framework: the Motion Capture Module (the process of recording movement); the Avatar Control Module (time-based transformations on the geometry and skeleton that comprises the avatar for animation purposes); and the Motion Map Module, which sits in between the other two modules (Direct and Indirect Mapping of motion capture data). For the purposes of our case study, this framework can be used to distinguish the kind of glitches or digital errors within each of the modules in the motion capture pipeline.

Georges Gagneré and Cédric Plessiet have recently proposed their AvatarStaging framework, which discusses both the scenic elements of their mixed reality set-up, and the different roles taken on by artists and technicians in this setting [4]. Their terminology for these roles is very useful: actor, mocaptor (performer being motion captured), digital artist (working on the avatar and 3D environment), manipulactor (assistant of digital artist and stage director guiding the mocaptor), and the stage director [4]. The AvatarStaging framework can be easily adapted to our contemporary dance setting, with minor modifications. It will be useful for discussing who is actually partaking, and in what way, in the exploration of glitch procedures.

Finally, amongst the extensive literature on visualisations of (dance) motion capture data, we consider Tsampounaris et al.'s work, in exploring visualisations in real-time motion capture, most relevant for the discussion of our case study [11]. These authors present visualisation variations of the same motion capture data, accessible through a custom-built UI. Most relevant for our discussion are their avatar variations and motion trail visualisations, in combination with a variety of viewpoints in the $3 \mathrm{D}$ environment.

\section{CASE STUDY: THE BEAUTIFUL GLITCH}

The case study we present here is based on the work of Rijmer during the artistic residency at STL. In the end of the artistic residency Rijmer presented a 20-minute long work-in-progress type of public performance. Entitled The Beautiful Glitch, this work was subdivided in two moments. The first part consisted of a live performance with the exploration of the motion capture system by two dancers. During the second part, a dance film was displayed, which was created from the recorded avatar visualisations during rehearsals, in post production. As the film was projected, one of the dancers was lying still on the stage in the dark, hardly perceptible; the other dancer had left the stage. Our analysis will first focus on the glitch experiences and procedures in the live motion capture set-up, and then address the choreographer's thinking, which also underlies the post production process of the dance film.

\subsection{Technical Set-Up}

Figure 1 shows a workflow diagram which describes the motion capture pipeline for both rehearsals and the final performance. Our motion capture system (The Captury) consists of hardware and software components, respectively Captury Live and Captury Studio. Captury Live acquires the motion capture data and our configuration was composed of eight networked RGB video cameras, mounted on a rig above the headspace of the dancers, covering an area of $6 \mathrm{mx} 6 \mathrm{~m}$. This markerless system allows to capture human motion of up to three performers (we tracked a maximum of two), based on colour contrast, at up to 120 frames per second. Captury Studio processes the video signals, handles the calibration process, creates a custom skeleton for each performer and can: 1) send video streams or motion data to a number of compatible software applications; and 2) record the data for post production. The Captury is usually employed in life sciences, sports, VR or entertainment scenarios, and was tested by our team in a mixed reality performance environment under very different lighting conditions.

Furthermore, figure 1 shows that we sent the skeleton data from Captury Studio to the Unity 3D engine to visualise one or two avatars in real-time. In Unity 3D, camera angles and perspectives on the avatars could be manipulated, and likewise, the relative position of the avatars to each other was determined. While we could have projected visualisations straight out of Unity 3D, we instead decided to add another element to our workflow. A video stream was sent from Unity 3D (via NDISyphon software) to Isadora, a versatile platform for programming live interactive audiovisual systems for performance and installations. Isadora enabled the research team to very quickly prototype multiple visualisations of the avatars through manipulations of the incoming video stream. In the beginning of the artistic residency, we also streamed motion data from the 12 skeletal points provided by the Captury Studio via the OSC protocol to Isadora, in order to generate abstract visualisations and/or use the OSC data to interactively manipulate the video stream. Later we abandoned the abstract method, with regards to the choreographer's intention to solely work with the avatar's human figure.

The motion capture pipeline described above was operated by one member of our research team who worked on two computers (PC1 and PC2). His role can be best defined as digital artist and operator (of the system), as shown in figure 2. A second researcher / digital artist (the first author) was responsible for further development of the avatar visualisations, and also took care of video projection design and mapping, using Isadora software on a third computer (PC3). The choreographer worked collaboratively with both digital artists, sharing her ideas and soliciting solutions. The final decision was always hers and thus her role can best be defined as stage director or artistic director. However, in working with the dancers, the choreographer was taking up an additional role, here described as 'manipulactor' (to borrow Gagneré and Plessiet's terminology [4]). As a choreographer, Rijmer devised improvisation tasks and developed the piece based on the dancer's input. Simultaneously, she needed to observe the avatar visualisations in their virtual environments and frequently had to instruct the dancers according to the needs of the digital artists, or according to her dramaturgical ideas. The dancers concentrated on their movement 


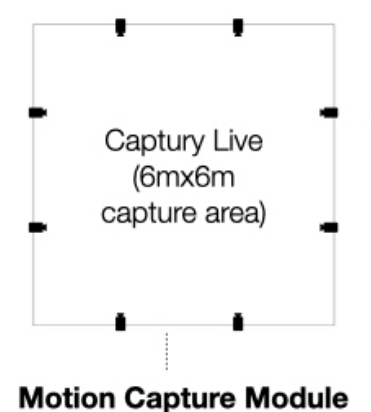

Motion Capture Module

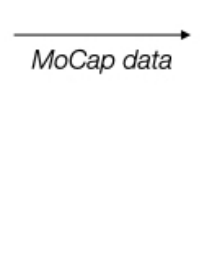

Motion Map Module

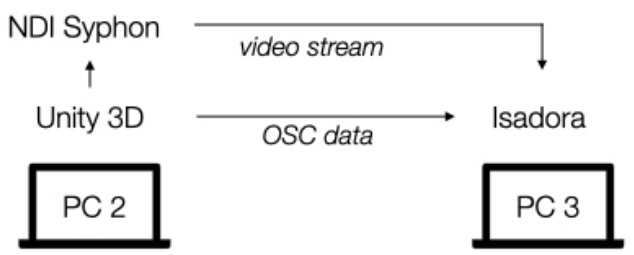

Avatar Control Module
Re-Visualisation Module

Figure 1: Workflow diagram of the motion capture pipeline.

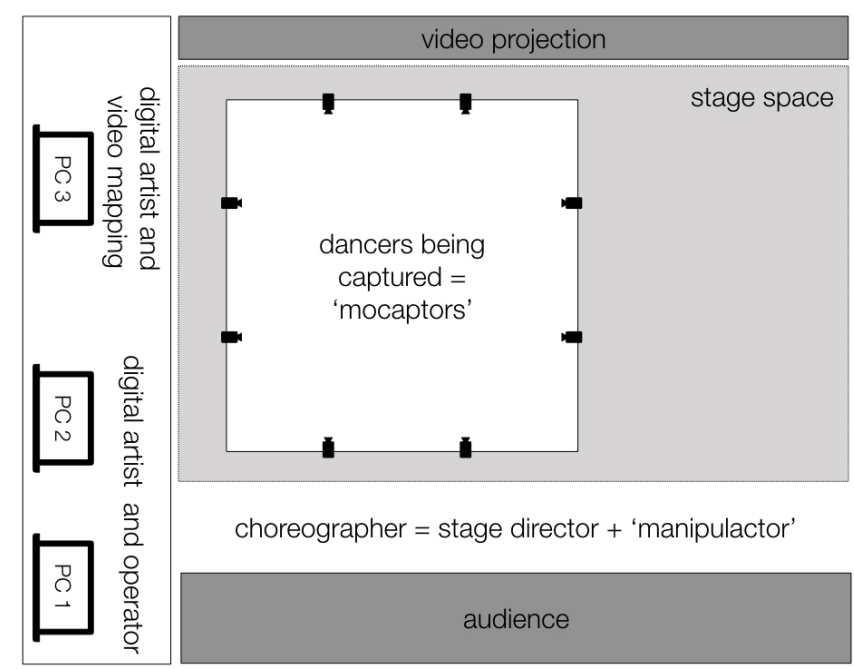

Figure 2: Roles of team members and staging configuration (floor plan).

explorations for the most part. Nevertheless, there were scenes in which they were also asked to be aware of their digital counterpart and manipulate the avatar's movement in real-time through their movement. In our discussion, we will come back to the roles of the artists and examine the impact of different forms of collaboration on the definition of tasks and responsibilities in this mixed reality performance environment.

\subsection{Choreographic Approach and Research}

Rijmer has developed the Body Logic Method (BLM) over the past years, which was described to us by the choreographer in interviews and follow-up conversations. BLM is a methodological framework consisting of two distinct modes of working: Dodging and Scanning. In the Dodging mode a number of improvisation and composition techniques are available, which can be explored individually, or combined in more complex situations. Working within the Dodging mode, the dancer focuses on redirecting body trajectories; reconfiguring body location; duplicating other dancers' body shape and orientation; redirecting other dancers' body trajectories through spatial blocking or physical touch; and applying dodging strategies between different parts of one's own body. When working in the Scanning mode, the dancers are 'listening' to inner flow states, impulses and become aware of their potentiality for movement in the moment to come. For example, a dancer should observe a particular place in the body from which movement initiates (prime mover), or become aware of a sequential movement pattern engaging multiple body parts. The Scanning mode then provides a number of focus points to enhance awareness, such as the respective exploration of stative, spatial, temporal and cognitive properties of the movement. Both Dodging and Scanning modes are training the dancer to improve deliberate choice making, movement creation and shaping the aesthetics of their performance. For Rijmer, "body logic" denotes the recognizable movement identity of a human being, as for example apparent in the unique way a person walks. Training in the BLM system enables the dancer to become aware of their idiosyncratic body logic and enhance the quality of the work relative to their experiential background and acquired knowledge.

To employ her Body Logic Method in a mixed reality performance environment within a real-time motion capture system represented a unique opportunity for Rijmer - to reflect on and augment her choreographic thinking and practice through the work with the digital tools. In the next section of the paper we examine how glitch procedures were articulated with the BLM techniques to a variety of avatar visualisations.

\subsection{A Typology of MoCap Failures}

The Captury can be a very stable motion capture system when set up in ideal conditions. In our experience with the system in the dance studio and on stage in suboptimal conditions, it can provoke digital failures more easily. To perform at the best possible level, The Captury needs to be set up in a well-lit space with homogeneous non-reflective wall and floor colour. Up to 12 cameras should be mounted at two different heights on a rig in the overhead space, not exceeding $3,50 \mathrm{~m}$. Alternatively, the system can be mounted on tripods of two different heights and surround the capture area, which can cover up to $8 \mathrm{mx} 8 \mathrm{~m}$. Performers should wear clothes which contrast well with the environment, skin colour of the performers and from each other. Consequently, clothes of different colours work well. The calibration of the system is achieved by using a custom calibration board, which is employed with each camera to get to the desired level of readings. Once the cameras 
are calibrated, a performer steps in the capture area and assumes a default pose (like the T-position for the Kinect sensor, only bending the forearms upwards into a kind of 'cactus' position). A skeleton is created, which corresponds to the morphology of the performer's body. Finally, the captured performer moves slowly exploring different directions, levels and turns to test the tracking.

We had experimented with the system previously on the occasion of a preparatory tech workshop and an introductory workshop with all participants of the Moving Digits project. During the artistic residency (which is subject of our analysis in this case study), we explored the limits of seven constituent elements in the system: light, colour and contrast, location of performer, movement, speed, occlusion, and interaction between performers.

These initial insights were methodically explored by Rijmer and became the basis for her glitch design procedures. This resulted in the following typology of MoCap failures:

- Light: Since our theatre lighting could be manually controlled, we tried different percentages to establish thresholds for stable tracking, for tracking difficulties (losing a few but not all skeleton points) and for losing the tracked performer

- Colour and contrast: We asked the dancers to bring several clothes and tried all kinds of colours. Again, we could observe colour (and contrast) combinations that allowed for stable tracking, and other combinations, which provoked errors such as: losing the tracking to different degrees, or confusing one tracked performer with another (and consequently attributing the wrong skeleton).

- Location of performer: Some (peripheral) areas within the motion capture space were potentially prone to error Dancers could explore these areas to provoke error, sometimes in combination with entrances and exits. When the tracking is at a stable level, the system could identify the performer and immediately attribute the skeleton. If the tracking did not work at ideal levels, a 'flickering' effect occurred, an alternation between a stable skeleton tracking (and representation) and the momentary loss (resulting in the distortion of the skeleton). Another spatial aspect was the level or plane in which the dancers moved: closer to the floor, the system had difficulties to track the dancer, or to distinguish two dancers moving close to each other. The resulting errors were distortions of the individual skeleton and impossible bodily positions of the avatars.

- Movement: As was to be expected, the dancer's movement posed all kinds of challenges to the motion capture system. As mentioned before, spatial location and planes influenced the quality of the tracking. Equally, the type of action (jump, turn, dislocation etc.) produced varying results. Turning or spinning around one's vertical axis often provoked confusion or loss of tracking.

- Speed: In addition, the speed of the dancer's movement had a substantial impact on the quality of the tracking. The simple formula here was: the faster the movement the more difficulties the system had in tracking the performers. Nevertheless, most of the time (after a partial loss or confusion in the tracking), the system was able to 'find' the dancer again
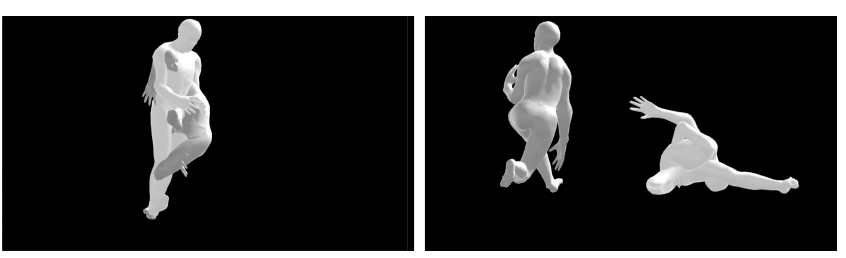

Figure 3: Examples of MoCap errors. Left: composite body. Right: one dancer is tracked well, the other is not.

and return to stable levels of tracking. Speed therefore was closely connected to timing and phrasing of the movement.

- Occlusion: In the case of a duet (or any set-up with more than one dancer), the system could not distinguish overlapping body spheres of the individuals. In consequence, the representations of the avatars in the duet started to bleed into each other and formed a bizarre composite body (see figure 3). Again, this could happen for short moments (flickering effect), or last for a longer time span. A different type of occlusions are self-occlusions, which can be provoked by the joining of the limbs, folding the body and so forth.

- Interaction between performers: Finally, the interaction between two dancers potentially multiplies the challenges for stable tracking, because all sources of digital errors mentioned above can occur in combinations. There are situations with strong potential that can be explored when one dancer is tracked well and the other is not.

\subsection{Designing Glitch Procedures and Subsequent Visualisation Strategies}

When we compare the list of digital errors provided by Kim Cascone [2] to the kinds of digital errors we have discovered in our motion capture pipeline (see Table 1), it becomes more evident in which ways Rijmer has explored digital failure to develop glitch procedures and visualisation strategies. The typology of MoCap failures presented above corresponds mostly to the category of "application errors" in Cascone's list, because the exploration of the limitations of our system provoke application errors. Following this logic, Cascone's "bugs" and "system crashes" can be linked to extreme exploration or violation of the system's limitations. Camera calibration can break down (for example, by changing a camera's position) requiring restart and re-calibration actions. However, these possibilities were not explored creatively by Rijmer. Distortion, Clipping and Aliasing techniques in Cascone's list can be related to specific tracking errors and mapping errors in our motion capture pipeline. Most significantly, glitches in our system become visible through the skeleton and subsequent avatar visualisations.

In order to develop (replicable) conditions for glitches to occur, our team used the methodical exploration of the system limitations (outlined in our typology of MoCap failures above) as our main source to design glitch procedures. More precisely, designing a glitch procedure in our mixed reality performance environment required designing an interaction strategy composed of selecting a 
Table 1: Correspondences between digital errors listed by Cascone [2] and discovered in our motion capture pipeline

\begin{tabular}{ll}
\hline $\begin{array}{l}\text { Kind of digital error } \\
\text { (Cascone) }\end{array}$ & Digital errors in the motion capture pipeline \\
\hline Glitch & $\begin{array}{l}\text { Glitches in our system become visible through the skeleton and avatar visualisations. } \\
\text { We consider the visual results from the application errors as our main source of glitches. }\end{array}$ \\
\hline Bugs & Very rarely, camera or performer calibration did not work at all (for no obvious reason). \\
\hline Application errors & $\begin{array}{l}\text { Application errors correspond to the limitations of our system, as described in our } \\
\text { typology of MoCap failures above (light, colour, performer location etc.). }\end{array}$ \\
\hline System crashes & $\begin{array}{l}\text { Camera calibration breaks down, the system has to be restarted and re-calibrated. } \\
\text { Performer calibration breaks down, re-calibration necessary. } \\
\text { Rarely, we experienced a system freeze (calibration was not possible). Consequence: re-starting the computer. }\end{array}$ \\
\hline Distortion & Refers in our case to the skeleton mapping and avatar visualisations. \\
\hline Clipping & Correspondence to partially missing skeleton data due to tracking errors. \\
\hline Aliasing & Corresponding to misidentification in the skeleton mapping process. \\
\hline Quantisation noise & Not applicable \\
\hline
\end{tabular}

specific threshold condition (within our typology of system limitations) in combination with an adequate choice of movement improvisation task to explore the condition. Designing glitch procedures is fundamentally different from designing glitches (see Menkman's critique of glitch art). In the context of mixed reality performance, designing glitch procedures means to design the intermedial condition in which specific types of glitches can occur without losing the key element of the unexpected and uncontrollable.

In another strand of Menkman's argument for a glitch studies approach [7], she states: "Glitch studies searches for the unfamiliar while at the same time it tries to de-familiarize the familiar." We consequently have analysed these elements in the work of Rijmer. Furthermore, we suggest extending these artistic strategies from the glitch procedures (in the motion capture phase) into what we call 're-visualisation module' (in the visualisation phase), as they correspond to different steps in the workflow (see figure 1). In other words, once the skeleton data were mapped onto an avatar in the Unity 3D engine, further possibilities presented themselves, which, adapting Menkman, can be designated as "search for the unfamiliar" and "strategies of de-familiarisation." Examples for the unfamiliar in Rijmer's visual approach include extreme and unusual camera angles and perspectives in the 3D space, such as a top view on the avatars, or the contrary, viewing the movement sequences from below. Strategies of de-familiarisation were employed both in the Unity $3 \mathrm{D}$ engine and the Isadora programming environment. For example, two avatars were placed so close to each other in Unity 3D that their bodies extended into each other, creating uncanny holes and bizarre composite bodies. In Isadora, alpha channels and masking techniques (see figure 4) were employed to reveal other imagery within the avatar body, or the figure was inserted in a virtual environment.

\section{DISCUSSION}

\subsection{Typology of MoCap Failures and Avatar Visualisation}

Our typology of MoCap failures builds on the work of Miller, who has identified errors in the markerless motion capture of human motion that occurred in the system used by him and his team, and has presented his artistic exploration of recorded 'bad captures' [8]. Errors mentioned by Miller included "not having enough cameras, improper stage lighting, and a relatively small stage size" [8]. Elsewhere in the paper, errors deriving from ambient light noise, occlusions and incorrect settings on camera exposure are mentioned. We have experienced the same errors in our MoCap system. Miller's error list can be linked to our typology of MoCap failures: these errors would fall into the categories of light, location of performer and occlusions. Building on Miller and our own observations, we have additionally experienced and described other error causes, including colour and contrast, movement, speed, and interaction between performers. Our typology of MoCap failures consists of a total of seven categories, which hopefully serve to be explored further by researchers and artists.

In the same paper Miller presents his visualisation approach of the recorded 'bad' motion capture data. In post-production, this data is used to experiment with an abstract aesthetic. Human motion data in Miller's work are transcoded into the visual dynamics of animated 3D modeled shapes, such as type and cloth simulations [8]. Contrary to this approach, Rijmer explores concrete avatars in the human form, both in real-time and edited in post-production. Since Rijmer was exploring the tensions between the biological and the digital body from the perspective of posthuman performance theory, she decided that working with a classic avatar in human form allowed her to visualise the glitch effects as a direct expression of her theme. Through recurring to a 'retro' aesthetic (the avatars she used have existed in our visual culture for more than twenty years) she could also successfully use artistic strategies of de-familiarisation in the re-visualisations of the avatar. Rijmer's perspectives on glitch 

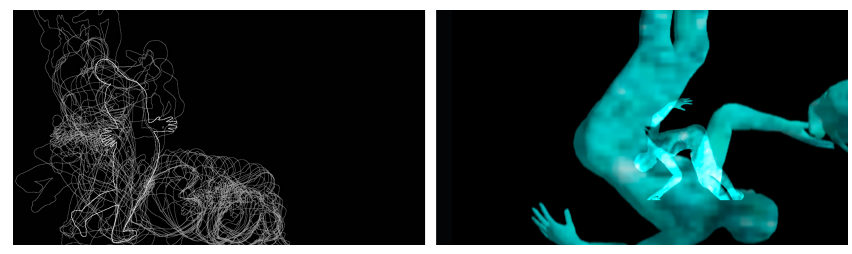

Figure 4: Re-visualisation examples in Isadora. Left: movement trails. Right: avatar figure mask.

and avatar, synthesised in our typology of MoCap failures and supported by our visualisation workflow, present pathways for other choreographers to explore.

\subsection{AvatarStaging Framework and Collaboration Models}

Our adaptation of the AvatarStaging framework by Gagneré and Plessiet [4] (see figure 2) for a dance case study brings two points to the discussion. Firstly, the team and team member's respective roles originally presented in this framework describe a setting, in which tasks are clearly defined and the process is highly determined. We have adapted the framework according to a different constitution of our (smaller) team, and the dissimilar form of collaboration (devising of improvisational tasks) chosen by Rijmer. In our adaptation, the roles of stage director and 'manipulactor' are combined in the work of the choreographer, who is the artistic director of the project and also directs the dancers with regard to the (interactive) visualisations. Secondly, and as a result of the different form of collaboration, the dancers (besides being the 'mocaptors') were involved in aspects of the re-visualisations, such as the positioning and resulting shapes of the avatars on the screen, or its movement characteristics and aesthetics. This resulted at times in a double role for the dancers who worked on the movement creation and partook in aspects of the digital artist's work. We conclude from the analysis of our adaptation of Gagneré and Plessiet's AvatarStaging framework, that their framework has much to offer methodologically, particularly when combined with a closer look to varying forms of collaboration in dance and theatre productions. These frequently result in the choice of diverse improvisational and compositional strategies and choices, which in turn allow for using distinct techniques in the motion capture and visualisation processes. We contribute to this framework with perspectives on how choreographers and dancers can relate to it - respectively, as 'manipulactors' and 're-visualisation collaborators'.

Our discussion regarding the influence of collaboration forms on the interpretation of roles in the avatar staging process can also be extended to the work of the digital artists. In figure 2 the team of Rijmer includes two digital artists. One is responsible for operating the motion capture system and avatar visualisation, while the other manipulates (re-visualises) the avatar visualisations and takes care of video mapping issues. However, in the field of re-visualisation strategies the work of both digital artists overlaps. Unusual camera perspectives and avatar positions are manipulated in Unity 3D (by digital artist 1), while video compositing and interactive manipulations are achieved in Isadora (by digital artist 2). This workflow and sharing of tasks within the same field (of creating re-visualisations) is a good example of the potential flexibility and fluidity of roles, when examined in the specific context of a given case study.

\subsection{Rapid Prototyping of Visualisations with MoCap Avatars}

Tsampounaris et al. have explored visualisations in real-time motion capture (for dance education) which use a custom-built user interface. In their software application, a variety of viewpoints in the 3D environment can be put to use, 3D avatar variations are available, and motion trail visualisations can be explored [11]. However, this software application cannot be adapted to the artist's needs in real-time, for example during rehearsal. In our case, Rijmer needed to enquire, on-the-fly, into visualisation possibilities that corresponded to her artistic vision. For her needs, the separation of 3D avatar visualisation in Unity 3D and the re-visualisation strategies programmed in Isadora allowed rapid prototyping of visual ideas in the rehearsal session of the artistic residency (see figure 5). The digital artists subsequently could develop these prototype visualisations for the public performance. In other words, our set-up (combining Unity 3D with Isadora) represented a more agile way of introducing substantial changes in the visuals. Once Rijmer was satisfied with the visuals, the video stream was recorded. During the residency Rijmer used the recorded video material to produce a dance film, which was shown to the audience in the second part of The Beautiful Glitch. This decoupling of avatar and re-revisualisation can provide an important strategy for those aiming for a flexible and adaptive workflow in real-time motion visualisation.

\subsection{Expanding MoCap Pipeline Model}

The glitch procedures which we introduced above can also be examined in the light of the motion capture pipeline model by Geigel and Schweppe [5]. Our typology of MoCap failures shows that glitches (and other errors) occur at the stages of motion capturing human motion (motion capture module) and the mapping process of the data (motion map module). In consequence, glitch procedures are designed by the artists during these two phases of the motion capture pipeline. In the case of Rijmer, movement improvisation strategies from her BLM system were combined with the exploration of a particular glitch type. On the contrary, data visualisation techniques fall into the last phase of Geigel and Schweppe's model (the avatar control module), and correspond to our work within the Unity 3D environment. Additionally, visualisation strategies developed for The Beautiful Glitch introduce the re-visualisation module in our workflow (see figure 1). By connecting the design of glitch procedures and the visualisation techniques to Geigel and Schweppe's model, researchers and artists can situate their work precisely in the respective phases of the motion capture process. Our inclusion of an additional module addresses a later stage, at which re-visualisations can be included in the artistic work with the glitch visualisations.

\section{CONCLUSION}

As referred in the introduction section, the main objective of Rijmer's project proposal was to specifically explore glitches, errors and failure working with the available motion capture system in 


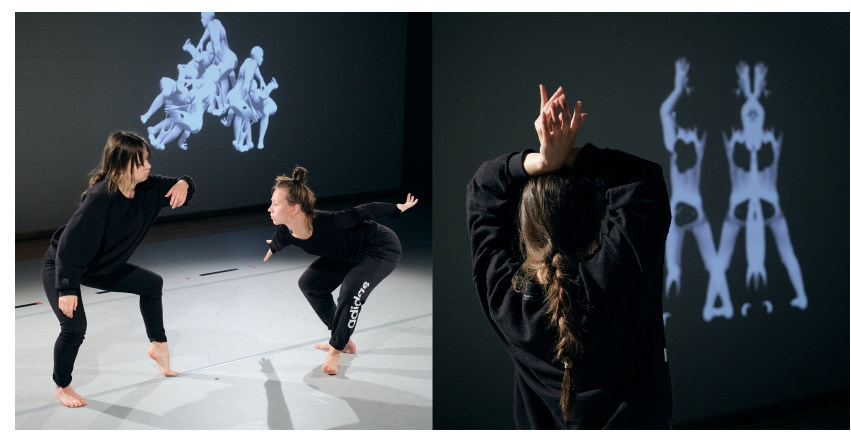

Figure 5: Rapid prototyping examples. Left: Multiplication of video streams in Isadora. Right: Unusual camera positioning in Unity $3 D$.

a mixed reality performance environment. Our case study shows how our research team adapted our technical set-up and collaboration methods to support the artist's vision and needs. In the discussion section, we have presented three contributions to build on the previous work existing in the field.

During the initial exploration of the motion capture pipeline, we tested the technical limits and the performance possibilities for the dancers within the MoCap system. This enquiry gave rise to our typology of MoCap failures, which can be used as well by other researchers who wish to work with the failures creatively. Moreover, the typology can also be used exclusively to test out what the limitations of a given system are. In our case, we have employed the typology to collaboratively design glitch procedures towards the choreographer's artistic goals. These glitch procedures combine strategies to provoke MoCap errors with improvisational tasks for the dancers to produce the real-time motion visualisations.

Early into the design of glitch procedures, we noticed that Rijmer was employing a disparate form of collaboration compared to the model presented in the AvatarStaging framework of Gagneré and Plessiet. In adapting their framework to contemporary dance, and specifically to our case study, we discovered that differing collaboration models affect the definition and description of roles taken on by team members, such as choreographer, dancers, digital artists and technicians. In consequence, our second contribution consists of the inclusion of collaboration models in the set-up and analysis of the avatar staging process.

Finally, our third contribution consists in the introduction of the 'rapid prototyping' workflow in mixed reality performance environments for rehearsals, residencies and other work-in-progress scenarios. This method prioritises the artistic vision within the time constraints of such creative process (in live performance contexts). In our case study, rapid prototyping was made possible through the decoupling of avatar and re-revisualisation, and resulted in designing a flexible workflow that combined several software tools (Unity 3D and Isadora, connected through NDISyphon).

Related to this third contribution, we expanded the MoCap pipeline model proposed by Geigel and Schweppe. We differentiated the motion capture data visualisation (the initial stage at which one sees a skeleton and/or avatar representing the captured motion) from the artistic visualisation approach and use of the motion capture data. We suggested calling the latter 're-visualisations.' From an audience point of view, only the re-visualisations will be seen. Whether abstract (as in Miller) or concrete (as in Rijmer), re-visualisations are materialisations of the artistic vision and thus different from initial motion capture data visualisation (which are habitually employed in non-artistic projects and research).

There are several research aspects of our case study that are beyond the scope of this paper and point at great potential for future research. One such research topic is movement understanding and movement analysis in mixed reality performance environment, where the dancers' movement could be described in a different way that takes the interdependencies and interrelatedness with other elements in the performance ecology into account. There is potential for the analysis of how choreographic thinking operates when extended into composing without the physical body. Finally, we want to look at how 3D MoCap visualisations can be experienced in VR preserving aspects of the live performance.

\section{ACKNOWLEDGMENTS}

We acknowledge the choreographer Sylvia Rijmer, dancers Hanna Junti and Liis Vares, Jochen Feitsch and the Moving Digits team. This research is co-funded by the Creative Europe programme of the EU (597398-CREA-1-2018-1-PT-CULT-COOP1). The authors acknowledge the support of LARSyS to this research (Projeto UIDB/50009/2020). The third author acknowledges ARDITI - Agência Regional para o Desenvolvimento e Tecnologia (project M142009-5369-FSE-000002 - PhD Studentship). We acknowledge ARDITI FDCTI-RAM 2018-reference:9, and FDCTI-RAM 2019-reference:8.

\section{REFERENCES}

[1] Michael Betancourt. 2016. Glitch Art in Theory and Practice: Critical Failures and Post-Digital Aesthetics. Focal Press.

[2] Kim Cascone. 2000. The Aesthetics of Failure: "Post-Digital" Tendencies in Contemporary Computer Music. Computer Music fournal 24, 4 (Dec. 2000), 12-18. https://doi.org/10.1162/014892600559489

[3] Paul Dourish. 2004. Where the action is: the foundations of embodied interaction. MIT press.

[4] Georges Gagneré and Cédric Plessiet. 2018. Experiencing avatar direction in low cost theatrical mixed reality setup. In Proceedings of the 5th International Conference on Movement and Computing (MOCO '18). ACM, Genoa, Italy, 1-6. https://doi.org/10.1145/3212721.3212892

[5] Joe Geigel and Marla Schweppe. 2011. Motion capture for realtime control of virtual actors in live, distributed, theatrical performances. In Face and Gesture 2011. 774-779. https://doi.org/10.1109/FG.2011.5771347 ISSN: null.

[6] Raul Masu, Nuno N. Correia, Stephan Jurgens, Ivana Druzetic, and William Primett. 2019. How do Dancers Want to Use Interactive Technology? Appropriation and Layers of Meaning Beyond Traditional Movement Mapping. In Proceedings of the 9th International Conference on Digital and Interactive Arts (ARTECH 2019). ACM, Braga, Portugal, 1-9. https://doi.org/10.1145/3359852.3359869

[7] Rosa Menkman. 2011. Glitch Studies Manifesto. In Video Vortex Reader II. Institute of Network Cultures. https://pdfs.semanticscholar.org/0732/ 56f0c90a712365890f379f7dc55bd1377f3e.pdf

[8] A. Bill Miller. 2015. Experimenting with Noise in Markerless Motion Capture. In Proceedings of the 2Nd International Workshop on Movement and Computing (MOCO '15). ACM, New York, NY, USA, 128-131. https://doi.org/10.1145/2790994. 2791019

[9] Mark Nunes. 2010. Error: Glitch, Noise, and Jam in New Media Cultures. Bloomsbury Publishing USA

[10] Chris Salter. 2010. Entangled: Technology and the Transformation of Performance. MIT Press.

[11] Georgios Tsampounaris, Katerina El Raheb, Vivi Katifori, and Yannis Ioannidis. 2016. Exploring Visualizations in Real-time Motion Capture for Dance Education. In Proceedings of the 20th Pan-Hellenic Conference on Informatics (PCI '16). ACM, Patras, Greece, 1-6. https://doi.org/10.1145/3003733.3003811 